\title{
EFEITO DO FÓSFORO EM CATALISADOR HZSM-5 PARA CONVERSÃO DE METANOL A PROPENO
}

\author{
R. B. DIAS ${ }^{1}$, L. TRAVALLONI ${ }^{1}$ e M. A. P. da SILVA $^{1}$ \\ ${ }^{1}$ Universidade Federal do Rio de Janeiro, Departamento de Engenharia Química \\ E-mail para contato: monica@eq.ufrj.br
}

\begin{abstract}
RESUMO - O propeno é uma importante matéria-prima petroquímica e sua demanda deve aumentar nos próximos anos. Devido a recentes descobertas de grandes reservas de gás natural, a conversão MTP (methanol to propylene) é uma alternativa atraente para a produção indireta de propeno. Um dos catalisadores mais usados na conversão MTP é a zeólita HZSM-5, cujo desempenho pode ser melhorado pela incorporação de elementos, como o fósforo. Neste trabalho, o desempenho da HZSM-5 e de sua modificação com P na conversão MTP foi avaliado. Diversos teores de P foram impregnados na HZSM-5 a partir de soluções de $\mathrm{H}_{3} \mathrm{PO}_{4}$. Os catalisadores obtidos foram caracterizados quanto a suas propriedades texturais e ácidas. Testes catalíticos foram realizados em diversas temperaturas e concentrações do reagente. A adição de P à HZSM-5 diminuiu sua área específica, volume de microporos, densidade de sítios ácidos e sua força. O catalisador com razão P/Al de 0,8 apresentou a maior estabilidade e produtividade de propeno.
\end{abstract}

\section{INTRODUÇÃO}

Olefinas leves são importantes blocos de construção na indústria petroquímica. O propeno é uma matéria-prima fundamental para a produção de várias commodities; dentre elas, plásticos de polipropileno, acrilonitrila e óxido de propileno. Espera-se um crescimento da demanda de propeno superior ao de sua oferta, o que, somado ao aumento da produção mundial de eteno, explica o maior preço do propeno em relação ao eteno em importantes mercados, como EUA e Ásia. Evidencia-se, portanto, a necessidade de estimular a produção do propeno e de buscar alternativas aos processos tradicionais de obtenção de olefinas leves, como o craqueamento a vapor e o craqueamento catalítico fluido de frações de petróleo bruto.

Em virtude das recentes descobertas de grandes reservas de gás de folhelho (shale gas), principalmente nos EUA, a petroquímica baseada no gás natural tem se fortalecido nos últimos anos, colaborando para suprir a crescente demanda de petroquímicos, em particular, poliolefinas. A reação MTO (methanol to olefins) tem ganhado interesse desde as duas crises do petróleo, na década de 70 , quando foi descoberta. Atualmente, há dois processos MTO comerciais: o primeiro foi desenvolvido pelas companhias UOP e Norsk Hydro, baseado em catalisadores de silicoaluminofosfato (SAPO-34), e o segundo, desenvolvido pela Lurgi, usa a zeólita HZSM-5 e também é conhecido como MTP (methanol to propylene). 


\section{9 a 22 de outubro de 2014 \\ Florianópolis/SC}

Uma série de catalisadores já foi testada na transformação do metanol a hidrocarbonetos, com destaque para a zeólita microporosa HZSM-5. Muitas formas de melhorar a estabilidade catalítica e o rendimento em olefinas leves foram investigadas, como modificações na estrutura do catalisador póssíntese. Sabe-se que a adição de fósforo à HZSM-5 é responsável pela diminuição da densidade e/ou da força de seus sítios ácidos mais fortes, associados à produção de compostos mais pesados. Dessa forma, muitos estudos vêm sendo realizados com o objetivo de entender a interação do fósforo com a estrutura zeolítica e de que forma esta é capaz de influenciar as propriedades catalíticas da reação MTO. Outro importante parâmetro que influencia a acidez do catalisador e, por conseguinte, a distribuição dos produtos de reação é a razão sílica-alumina (SAR) da zeólita. Diferenças na síntese do catalisador podem significar maiores ou menores densidades de sítios ácidos.

Este trabalho teve como objetivo geral avaliar o desempenho da HZSM-5 e da HZSM-5 modificada por fósforo na conversão de metanol a olefinas leves, especialmente propeno. O desempenho dos catalisadores com diversos teores de fósforo foi comparado, analisando-se a influência da temperatura e da pressão parcial do reagente. Buscou-se o teor ótimo de fósforo no catalisador, bem como as condições que maximizassem o rendimento no produto de interesse. Por último, avaliou-se a influência da razão SAR da zeólita na performance catalítica.

\section{METODOLOGIA}

\subsection{Preparo dos Catalisadores}

A incorporação de fósforo à HZSM-5 comercial $(\mathrm{SAR}=24)$ foi efetuada por impregnação úmida com solução aquosa de $\mathrm{H}_{3} \mathrm{PO}_{4}$ (Vetec, PA). Após a adição da solução à zeólita, a suspensão foi agitada por $18 \mathrm{~h}$ à temperatura ambiente e, em seguida, foi seca a $70^{\circ} \mathrm{C}$ sob vácuo. $\mathrm{O}$ sólido obtido foi seco em estufa a $100{ }^{\circ} \mathrm{C}$ por $18 \mathrm{~h}$ e então calcinado a $550{ }^{\circ} \mathrm{C}$. A nomenclatura dos catalisadores com fósforo foi xPHZSM-5, sendo x o teor de fósforo (\% m/m). Empregou-se, ainda, outra zeólita, com maior SAR e sem adição de fósforo, denominada HZSM-5 (41).

\subsection{Caracterização dos Catalisadores}

Análises de fluorescência de raios-X (FRX) foram realizadas num espectrômetro Rigaku, modelo Rix 3100, com tubo de ródio $(4 \mathrm{~kW})$. Difratogramas de raios-X foram obtidos num aparelho Rigaku Miniflex (30 kV, $15 \mathrm{~mA}$ ); a varredura foi realizada de 2 a $60^{\circ}$, em intervalos de $0,05^{\circ} / \mathrm{s}$. Para a determinação das propriedades texturais, foram feitas medidas de adsorção física de $\mathrm{N}_{2}$ a $-196{ }^{\circ} \mathrm{C}$ em amostras pré-tratadas sob vácuo a $300{ }^{\circ} \mathrm{C}$ por $18 \mathrm{~h}$, num equipamento TriStar 3000 da Micromeritics. Análises de decomposição de n-propilamina a temperatura programada (TPDpropilamina) foram realizadas num equipamento TPD/TPR 2900 da Micromeritics acoplado a um espectrômetro de massa Pfeiffer, modelo Omnistar 422; as amostras foram aquecidas até $500{ }^{\circ} \mathrm{C}$ $\left(5^{\circ} \mathrm{C} / \mathrm{min}\right)$ e esta temperatura foi mantida por $2 \mathrm{~h}$.

\subsection{Testes Catalíticos}

Testes catalíticos foram realizados em reator tubular de leito fixo operando à pressão 
atmosférica. Antes de cada teste, os catalisadores foram submetidos a um tratamento in situ a $500{ }^{\circ} \mathrm{C}$ $\left(10^{\circ} \mathrm{C} / \mathrm{min}\right)$ por $1 \mathrm{~h}$, sob corrente de He de $30 \mathrm{~mL} / \mathrm{min}$. O metanol foi alimentado à unidade por uma bomba seringa (BBRAUN) e vaporizado a $200^{\circ} \mathrm{C}$, empregando $\mathrm{He}$ como gás de arraste. O efluente do reator foi analisado em linha por um cromatógrafo HP 6890, dotado de coluna PLOT Q e detectores de ionização por chama e de condutividade térmica. A reação foi realizada a $350-600{ }^{\circ} \mathrm{C}$, com pressão parcial de metanol de 0,12-0,36 atm. Também foram avaliados os efeitos do tempo de reação, do teor de fósforo e da SAR da zeólita em sua atividade e estabilidade.

\section{RESULTADOS E DISCUSSÃO}

\subsection{Caracterização dos Catalisadores}

A Tabela 1 apresenta as composições químicas obtidas por FRX, bem como as razões molares $\mathrm{SiO}_{2} / \mathrm{Al}_{2} \mathrm{O}_{3}$ (SAR) e $\mathrm{P}_{2} \mathrm{O}_{5} / \mathrm{Al}_{2} \mathrm{O}_{3}$ dos catalisadores. As propriedades texturais são apresentadas na Tabela 2. A área específica e o volume de microporos decresceram linearmente $\left(R^{2}=0,99\right)$ com a adição de fósforo, o que está de acordo com a literatura (Barros et al., 2007).

Tabela 1 - Composição química dos catalisadores.

\begin{tabular}{cccccccc}
\hline Catalisador & $\begin{array}{c}\mathbf{S i O}_{\mathbf{2}} \\
(\mathbf{\%} \mathbf{m} / \mathbf{m})\end{array}$ & $\begin{array}{c}\mathbf{A l}_{\mathbf{2}} \mathbf{O}_{\mathbf{3}} \\
(\mathbf{\%} \mathbf{m} / \mathbf{m})\end{array}$ & $\begin{array}{c}\mathbf{P}_{\mathbf{2}} \mathbf{O}_{\mathbf{5}} \\
(\mathbf{\%} \mathbf{m} / \mathbf{m})\end{array}$ & $\begin{array}{c}\mathbf{P} \\
(\boldsymbol{\%} \mathbf{m} / \mathbf{m})\end{array}$ & $\begin{array}{c}\mathbf{A l} \\
(\mathbf{\%} \mathbf{m} / \mathbf{m})\end{array}$ & $\begin{array}{c}\mathbf{S i O}_{2} / \mathbf{A l}_{\mathbf{2}} \mathbf{O}_{\mathbf{3}} \\
(\mathbf{m o l} / \mathbf{m o l})\end{array}$ & $\begin{array}{c}\mathbf{P}_{2} \mathbf{O}_{\mathbf{5}} / \mathbf{A l}_{\mathbf{2}} \mathbf{O}_{\mathbf{3}} \\
(\mathbf{m o l} / \mathbf{m o l})\end{array}$ \\
\hline HZSM-5 & 93,3 & 6,7 & 0,0 & 0,0 & 3,8 & 23,8 & 0,0 \\
1,2PHZSM-5 & 90,7 & 6,4 & 2,9 & 1,2 & 3,6 & 24,0 & 0,3 \\
3,2PHZSM-5 & 86,6 & 6,2 & 7,3 & 3,2 & 3,5 & 23,9 & 0,8 \\
5,0PHZSM-5 & 82,6 & 5,9 & 11,5 & 5,0 & 3,3 & 23,7 & 1,3 \\
HZSM-5 (41) & 95,8 & 4,0 & 0,0 & 0,0 & 2,1 & 41,2 & 0,0 \\
\hline
\end{tabular}

Tabela 2 - Propriedades texturais dos catalisadores.

\begin{tabular}{cccc}
\hline Catalisador & $\begin{array}{c}\text { Área específica } \\
\left(\mathbf{m}^{\mathbf{2}} \mathbf{/ g}\right)^{\mathbf{a}}\end{array}$ & $\begin{array}{c}\text { Volume de } \\
\mathbf{m i c r o p o r o s}\left(\mathbf{c m}^{\mathbf{3}} / \mathbf{g}\right)\end{array}$ & $\begin{array}{c}\text { Volume de } \\
\mathbf{m e s o p o r o s}\left(\mathbf{c m}^{\mathbf{3}} / \mathbf{g}\right)\end{array}$ \\
\hline HZSM-5 & 370 & 0,13 & 0,030 \\
1,2PHZSM-5 & 340 & 0,11 & 0,030 \\
3,2PHZSM-5 & 253 & 0,09 & 0,029 \\
5,0PHZSM-5 & 165 & 0,07 & 0,013 \\
\hline \multicolumn{4}{c}{${ }^{a}$ método BET; ${ }^{b}$ método t-plot; ${ }^{\text {c }}$ método BJH. }
\end{tabular}

A Figura 1 ilustra os difratogramas dos catalisadores, que apresentaram três intensos picos característicos, situados em $2 \theta=23-24^{\circ}$, confirmando uma estrutura do tipo MFI (Lu et al., 2003). Os difratogramas não apresentaram picos largos, correspondentes a fases amorfas, o que indica a ausência de impurezas detectáveis. A adição de fósforo não alterou a estrutura MFI da HZSM-5, mas diminuiu o grau de cristalinidade em teores acima de $3,2 \% \mathrm{~m} / \mathrm{m}$, o que é evidenciado pela redução na intensidade dos picos característicos da estrutura (Barros et al., 2007), bem como dos picos a $2 \theta \approx 8^{\circ}$. 


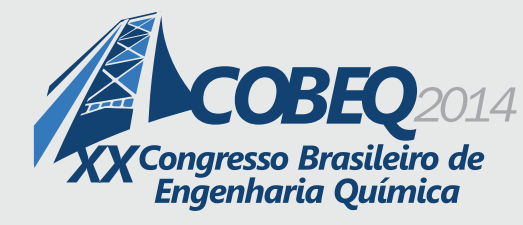

19 a 22 de outubro de 2014
Florianópolis/SC

Os resultados de TPD-propilamina são apresentados na Figura 2. A formação de propeno ocorre em sítios ácidos de Brønsted. A adição de fósforo diminuiu de forma significativa a dessorção de propeno e, consequentemente, a densidade de sítios de Brønsted, o que está de acordo com a literatura (Caeiro et al., 2006). Embora não haja um consenso, estudos indicam que a reação MTO ocorre sobre sítios ácidos de Brønsted (Støcker et al., 1999).

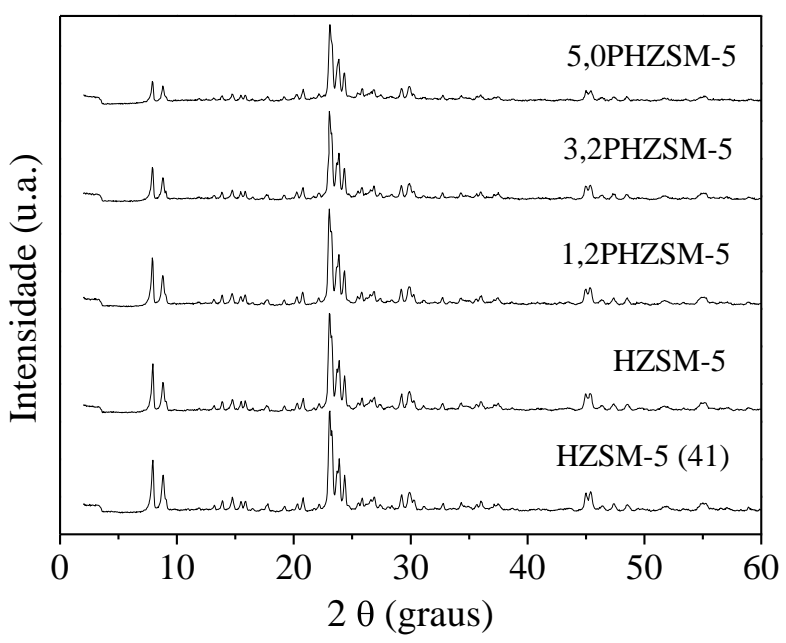

Figura 1 - Difratogramas dos catalisadores.

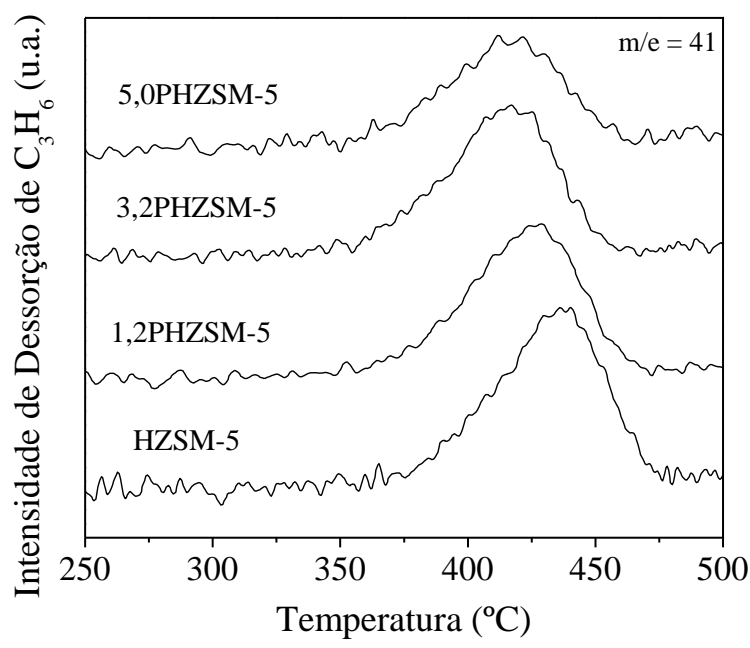

Figura 2 - Perfis de dessorção de propeno.

\subsection{Testes Catalíticos}

A Figura 3 apresenta a distribuição de produtos em função do tempo reacional para os catalisadores estudados. Para HZSM-5 e 1,2PHZSM-5, houve formação significativa de DME a partir de 157 min de reação. O catalisador 3,2PHZSM-5 apresentou elevada estabilidade, sem alterações significativas na distribuição de produtos ao longo do tempo. Como este catalisador possui menor densidade de sítios ácidos, responsáveis pela formação de aromáticos, baixos rendimentos em $\mathrm{C}_{6}{ }^{+}$já eram esperados - resultado também obtido para o 5,0PHZSM-5. Portanto, para teores de fósforo abaixo de 3,2\%, houve desativação catalítica pela formação de aromáticos e, por conseguinte, de coque (Bjørgen et al., 2003). Além disso, para o maior teor de fósforo, verificou-se a formação de DME desde o início da reação, o que sugere uma acidez excessivamente baixa ou um bloqueio parcial dos poros do catalisador por espécies fosfóricas (Lee et al., 2009), indicado pelos resultados de adsorção de $\mathrm{N}_{2}$.

As Figuras 4 e 5 ilustram a influência do teor de fósforo na conversão de metanol e no rendimento de propeno, empregando 0,12 atm de metanol e $500{ }^{\circ} \mathrm{C}$. A maior produtividade de propeno foi obtida para 3,2\% de fósforo. A adição de fósforo diminui a acidez do catalisador (Lee et al., 2009), dificultando a formação de compostos mais pesados e coque. Com isso, nota-se a maior estabilidade do catalisador frente à desativação, o que explica a conversão completa do reagente durante toda a reação. No caso do catalisador 5,0PHZSM-5, as menores produtividade de propeno e conversão de metanol em relação ao 3,2PHZSM-5 podem ser associadas à diminuição excessiva da acidez, dificultando a formação de hidrocarbonetos e favorecendo a formação de DME, apenas. 

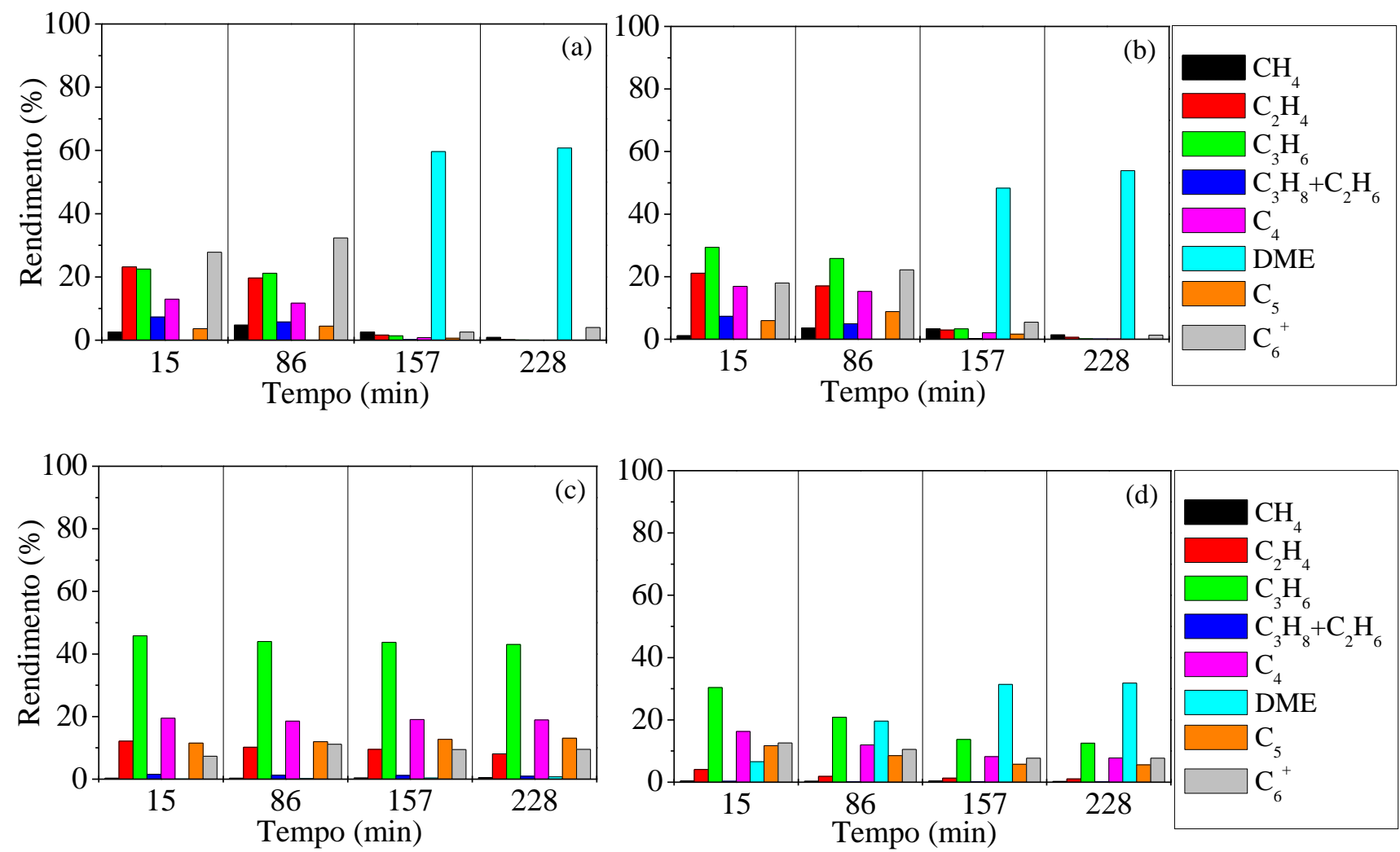

Figura 3 - Distribuição de produtos em função do tempo de reação, usando 0,12 atm de metanol, $500{ }^{\circ} \mathrm{C}$ e (a) HZSM-5, (b) 1,2PHZSM-5, (c) 3,2PHZSM-5, (d) 5,0PHZSM-5.

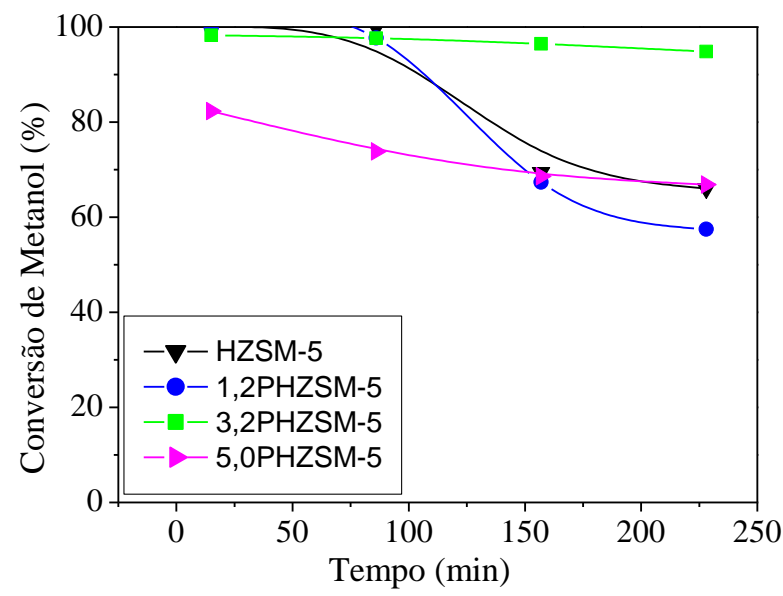

Figura 4 - Conversão de metanol em função do teor de fósforo.

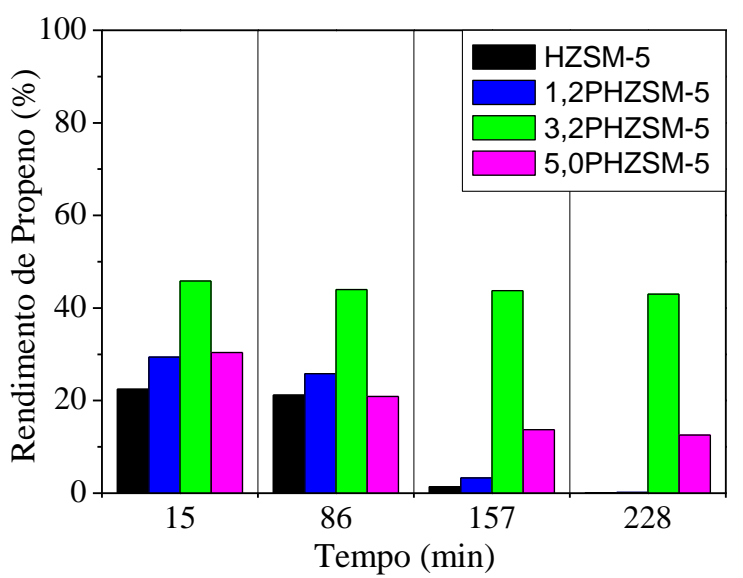

Figura 5 - Rendimento de propeno em função do teor de fósforo. 
O efeito da pressão parcial de metanol na produtividade de propeno e na conversão do reagente é apresentado nas Figuras 6 e 7, a $500{ }^{\circ} \mathrm{C}$ e empregando 3,2PHZSM-5. Para a maior pressão de metanol, verificou-se uma maior desativação do catalisador, como esperado. A literatura reporta que a formação de olefinas leves é favorecida a baixas pressões parciais de metanol (Dehertog e Froment, 1991). Neste trabalho, entretanto, observou-se uma menor produtividade de propeno para a menor pressão de metanol ao longo do tempo de reação avaliado. Porém, nota-se que a produtividade de propeno diminuiu rapidamente para a maior pressão de metanol.

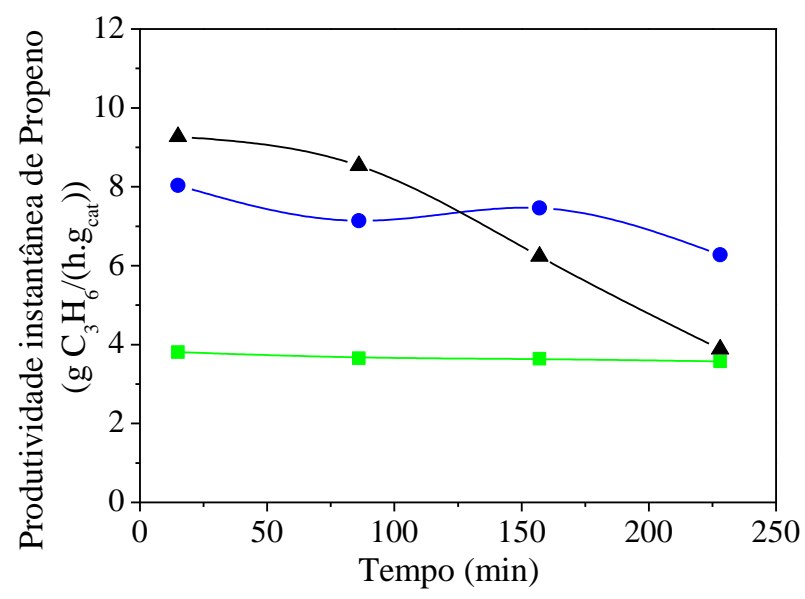

Figura 6 - Produtividade de propeno em função da pressão parcial de metanol.

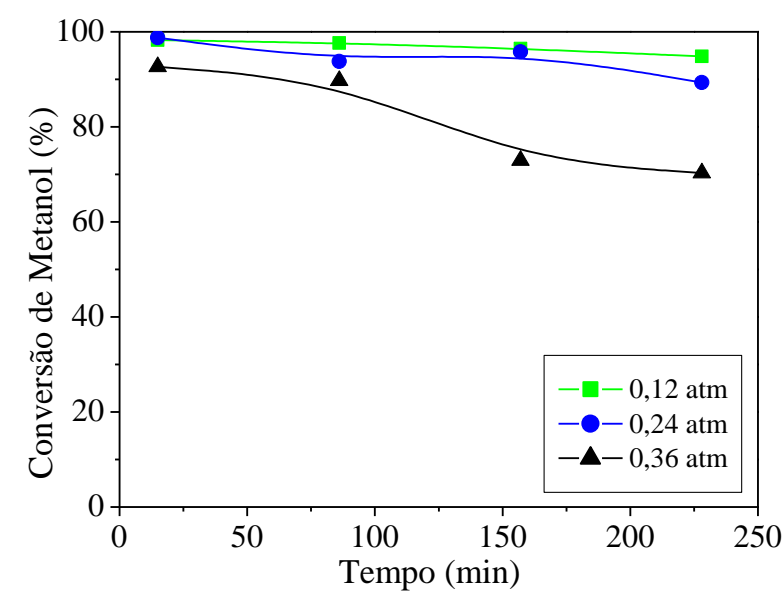

Figura 7 - Conversão de metanol em função da pressão parcial de metanol.

A Figura 8 apresenta a distribuição de produtos em função da temperatura, no primeiro (15 min) e no último (228 min) tempos reacionais, empregando HZSM-5 (a; b) e 3,2PHZSM-5 (c; d). Percebese a elevada estabilidade da 3,2PHZSM-5, caracterizada pela similaridade de seus perfis, assim como seu maior rendimento em propeno durante toda a reação. A zeólita comercial desativou em maiores temperaturas, conforme evidenciado pelos altos rendimentos em DME a 450 e $500{ }^{\circ} \mathrm{C}$. Para ambos os catalisadores, nota-se a existência de uma temperatura ótima. A conversão de metanol é desfavorecida a baixas temperaturas, enquanto a formação de coque e a desativação são maiores a altas temperaturas (Chen et al., 2000).

A influência da SAR da zeólita na conversão de metanol e no rendimento de propeno, empregando 0,12 atm de metanol e $500{ }^{\circ} \mathrm{C}$, é ilustrada nas Figuras 9 e 10. Houve uma desativação significativa da HZSM-5 com SAR = 24, enquanto a conversão permaneceu em $100 \%$ durante toda a reação para a HZSM-5 (41). Percebe-se um elevado rendimento em $\mathrm{C}_{6}{ }^{+}$para a HZSM-5 (24) em 15 min de reação e, em decorrência da desativação, uma maior formação de DME no final da reação. Os perfis da HZSM-5 (41) ilustram sua elevada estabilidade e maior rendimento de propeno. A literatura aponta que reações de formação de aromáticos são suprimidas ao se empregar catalisadores com maiores SAR (Dehertog e Froment, 1991). 

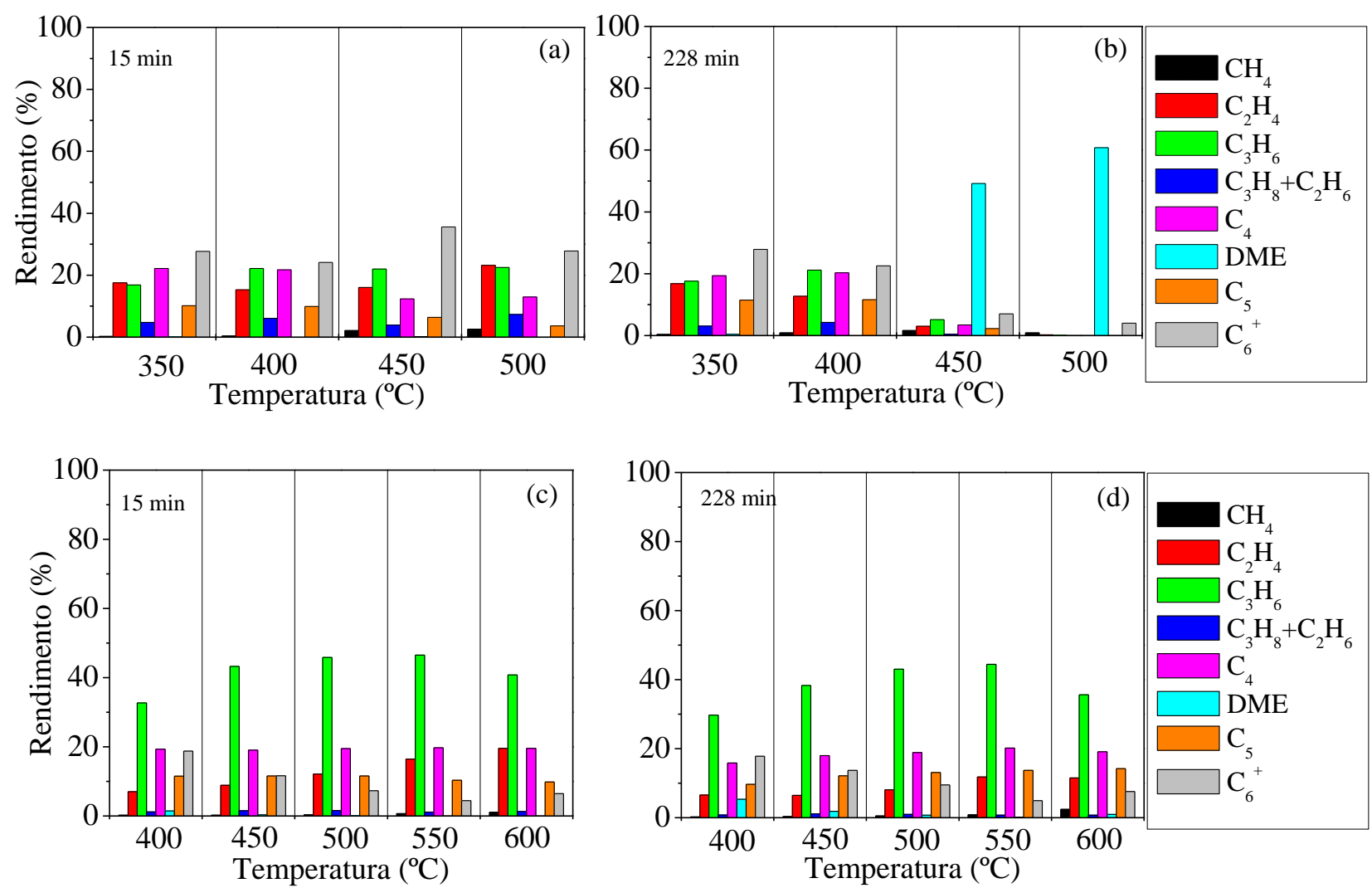

Figura 8 - Distribuição de produtos em função da temperatura, usando 0,12 atm de metanol; (a) HZSM-5, 15 min; (b) HZSM-5, 228 min; (c) 3,2PHZSM-5, 15 min; (d) 3,2PHZSM-5, 228 min.

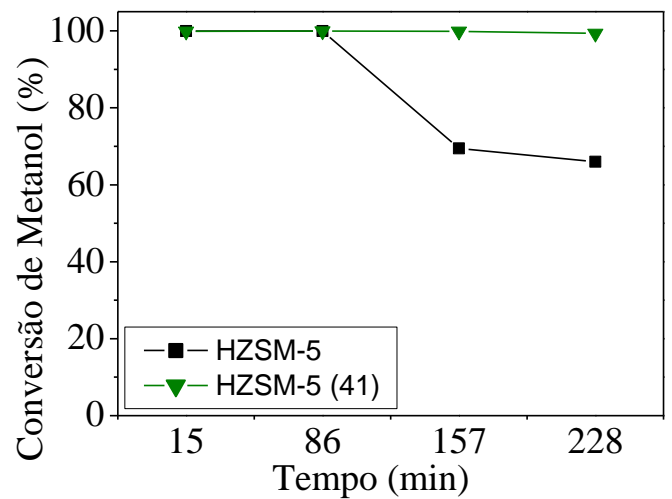

Figura 9 - Conversão de metanol em função da razão SAR.

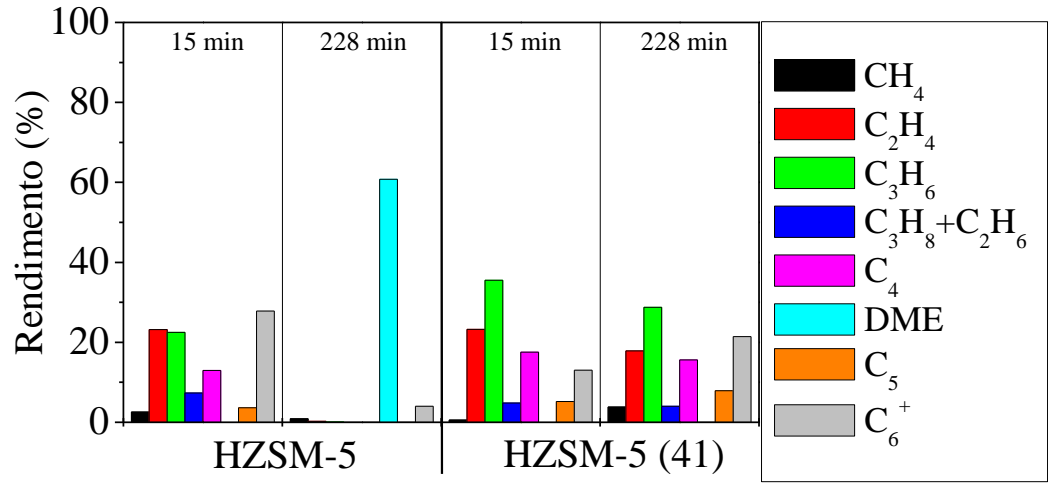

Figura 10 - Distribuição de produtos em função da razão SAR, a 15 e 228 min de reação. 


\section{CONCLUSÕES}

A desativação da HZSM-5 comercial se deve à formação de aromáticos e coque sobre os sítios ácidos mais fortes. A adição de fósforo diminuiu a concentração de sítios ácidos de Brønsted, a área específica e o volume de microporos do catalisador. As espécies de fósforo são responsáveis pelo bloqueio parcial dos poros da zeólita, o que explica o menor rendimento em propeno do catalisador com o maior teor de fósforo. O catalisador 3,2PHZSM-5 (razão P/Al = 0,8) apresentou a maior formação de propeno e a maior estabilidade. A produtividade de propeno no início da reação foi maior para a maior pressão parcial de metanol, porém diminuiu significativamente ao longo do tempo. A formação de propeno com o catalisador 3,2PHZSM-5 foi máxima a $500-550{ }^{\circ} \mathrm{C}$, cerca de $100{ }^{\circ} \mathrm{C}$ acima da temperatura ótima para a HZSM-5. O rendimento em propeno foi maior para a maior razão SAR, enquanto a formação de $\mathrm{C}_{6}{ }^{+}$foi mais elevada para a zeólita de menor SAR.

\section{AGRADECIMENTOS}

Ao NUCAT/PEQ/COPPE/UFRJ e ao GreenTec/EQ/UFRJ pelas análises de caracterização dos catalisadores; ao CENPES/Petrobras pelo fornecimento das zeólitas; à ANP, à FINEP, ao CNPq e ao PRH-13 pelo apoio financeiro.

\section{REFERÊNCIAS}

BARROS, Z. S; ZOTIN, F. M. Z.; HENRIQUES, C. A. Conversion of natural gas to higher valued products: light olefins production from methanol over ZSM-5 zeolites. Stud. Surf. Sci. Catal., v. 167, p. 255-260, 2007.

BJØRGEN, M.; OLSBYE, U.; KOLBOE, S. Coke precursor formation and zeolite deactivation: mechanistic insights from hexamethylbenzene conversion. J. Catal., v. 215, p. 30-44, 2003.

CAEIRO, G.; MAGNOUX, P.; LOPES, J. M.; RIBEIRO, F. R.; MENEZES, S. M. C.; COSTA, A. F.; CERQUEIRA, H. S. Stabilization effect of phosphorus on steamed H-MFI zeolites. Appl. Catal. A, v. 314, p. 160-171, 2006.

CHEN, D.; REBO, H. P.; GRØNVOLD, A.; MOLJORD, K.; HOLMEN, A. Methanol conversion to light olefins over SAPO-34: kinetic modeling of coke formation. Micropor. Mesopor. Mater., v. 3536, p. 121-135, 2000.

DEHERTOG, W. J. H.; FROMENT, G.F. Production of light alkenes from methanol on ZSM-5 catalysts. Appl. Catal., v. 71, p. 153-165, 1991.

LEE, Y.-J.; KIM, J. M.; BAE, J. W.; SHIN, C.-H.; JUN, K.-W. Phosphorus induced hydrothermal stability and enhanced catalytic activity of ZSM-5 in methanol to DME conversion. Fuel, v. 88, p. 1915-1921, 2009.

LU, R.; TANGBO, H.; WANG, Q.; XIANG, S. Properties and characterization of modified HZSM-5 zeolites. J. Natural Gas Chem., v. 12, p. 56-62, 2003.

STØCKER, M. Methanol-to-hydrocarbons: catalytic materials and their behavior. Micropor. Mesopor. Mater., v. 29, p. 3-48, 1999. 